\title{
The consequences of disclosing key audit matters (KAMs): A review of the academic literature
}

\author{
Anna Gold, Melina Heilmann
}

Received 3 September 2018 | Accepted 17 December $2018 \quad$ Published 11 March 2019

\begin{abstract}
Recent years have witnessed a change in the auditor reporting model. One of these developments is the auditor's issuance of so-called Key Audit Matters in the auditor's report, where they disclose "those matters that, in the auditor's professional judgment, were of most significance in the audit of the financial statements of the current period". In this paper, we review the emerging body of academic research which examines the effects of KAM disclosures in the auditor's report. We investigate research that has examined the effect of KAM disclosures on (1) investor behavior and market reaction, (2) auditor responses, (3) auditor liability, and (4) client management responses. The objective of this paper is to provide an overview of the existing literature and to summarize the preliminary findings and implications of 22 studies.
\end{abstract}

\section{Keywords}

Key audit matters, auditor reporting model, stakeholder responses

\section{Practical relevance}

This literature review is of interest to auditors, standard setters, investors, regulators, and other stakeholders affected by auditor's reporting, as the disclosure of KAMs significantly changes auditors' communication. Readers will gain insights into initial research findings on KAM disclosure that will help evaluate the consequences of the new reporting requirements.

\section{Introduction}

The auditor's report is the primary mean of communication between auditors and financial statement users (PCAOB 2017). However, the traditional reporting model is highly standardized and therefore frequently perceived as insufficiently useful, informative, and transparent (Asare and Wright 2012; Church et al. 2008; IAASB 2011). In particular, in the aftermath of the financial crisis of 2008, regulators, standard setters and the investment community started seriously questioning the informative value of the auditor's report. Similarly, academic research in the last decade has also repeatedly emphasized that there may be a need for change, motivating audit re- port reforms. For example, Carcello (2012) and Turner et al. (2010) find that users generally valued the auditor's opinion but showed little interest in reading the actual report given its highly standardized format. Users assessed the traditional auditor's report as uninformative in particular because nearly all public companies receive the same unqualified opinion (Church et al. 2008; Gray et al. 2011). In their research synthesis, Mock et al. (2013) conclude that stakeholders desire more information about the audit, the auditor and financial statements. Moreover, Vanstraelen et al. (2012) find that users were interested in additional disclosures on audit findings such as key areas of risks. Overall, research results indicate that there is a gap between information that users desire about 
financial statements and the audit and what is available through a company's audited financial statements and the auditor's report. Standard setters and researchers refer to this phenomenon as the "information gap" (IAASB 2012; Mock et al. 2013). In 2012, the chairman of the IAASB stressed that "more than ever before, $[\ldots]$ users of the audited financial statements are calling for more pertinent information for their decision-making in today's global business environment with increasingly complex financial reporting requirements" (IAASB 2012). The information gap is closely related to the long-standing expectation gap which describes the difference between users' expectations of an audit and what an audit actually is (IAASB 2011). As academic research provides ample of evidence on the persistence of this gap (e.g., Chong and Pflugrath 2008; Gold et al. 2012), Gray et al. (2011) argue that there is a need to make significant changes to the auditor's report in order to reduce misperceptions.

These debates and research findings have resulted in multiple initiatives across the globe to enhance the communicative value of the auditor's report. The International Auditing and Assurance Standards Board (IAASB), the European Commission (EC), the Public Company Accounting Oversight Board (PCAOB), and the U.K. Financial Reporting Council (FRC) finalized their projects to enhance the auditor's report. One of the most significant amendments is the disclosure of Key Audit Matters (KAMs) or Critical Audit Matters (CAMs, which are the equivalent concept in the U.S. jurisdiction) in the auditor's report. According to ISA 701.8, key audit matters are "those matters that, in the auditor's professional judgment, were of most significance in the audit of the financial statements of the current period". Unlike the traditional auditor's report, the revised form allows for more customized information disclosed about the client- and engagement-specific observations made by the auditor. As such, the primary objective of standard setters and regulators is the transformation of the traditional pass/fail-model into a more individual and valuable report in order to meet the informational needs of financial statement users.

In this paper, we review the emerging body of academic research which examines the effects of KAM disclosures in the auditor's report. We do so by examining four distinct streams of research. First, we review research papers that investigate whether KAM disclosures indeed have the potential of meeting the expectations of standard setters and regulators with respect to providing a more valuable reporting model. Second, some scholars argue that the introduction of the KAM section in the auditor's report may not only influence financial statement users' perception and decisions but could also have an influence on the audit itself. For example, Reid et al. (2018) suggest that auditors may exert more effort during the audit because of an increased sense of accountability due to anticipation of KAM disclosure. Similarly, the IAASB (2015b) refers to such potential increases of auditor professional skepticism in areas where KAMs are identified and, as a result, increased audit quality, not only in the perception of the users.
As a result of the potential of audit quality implications, a second stream of research examines auditor responses to KAM disclosures. Third, in the course of the development of the new reporting requirements, auditor legal liability was a frequently debated controversy, particularly in the United States (e.g., Tysiac 2013). The concern is that disclosing KAMs might increase jurors' perceptions of auditor liability, especially when auditors have failed to detect misstatements. Hence, a third stream of literature examines the effects of KAM disclosure on auditor's liability. Fourth, there is a burgeoning literature on client management reporting behavior in response to KAM disclosure. A potential benefit of KAM disclosures in this area is that client management may adopt less aggressive accounting in anticipation of auditor disclosure (Reid et al. 2018). The IAASB (2015) also referred to increased attention by management to KAM disclosures, which could have an indirect, beneficial effect on reporting behavior.

The objective of this paper is to provide an overview of the existing literature and to summarize the preliminary findings and implications of 22 studies. $^{1}$ In our review, we include research studies available until 1 August 2018. Since research in this area is in its infancy, we include working papers in our review rather than focusing exclusively on published articles.

The remainder of this paper is organized as follows. In the next section, we provide a brief overview of related regulatory developments. Section 3 summarizes and discusses the results of academic studies on KAM disclosures. The final section presents our conclusions including implications for future research and for the audit profession.

\section{Regulatory background}

In May 2011, the IAASB published the consultation paper "Enhancing the value of auditor reporting: Exploring options for change", discussing possible ways to improve the auditor's report, particularly regarding the increased need for information by users and the persisting expectation and information gap (IAASB 2011). The IAASB's (2012) Invitation to Comment resulted in a high level of support by various stakeholders for the amendments proposed by the IAASB (Prasad and Chand 2017; Simnett and Huggins 2014). In July 2013, these initiatives were followed by the Exposure Draft "Reporting on audited financial statements: Proposed new and revised International Standards on Auditing (ISAs)" (IAASB 2013). On 15 January 2015, the IAASB concluded its project with the release of the final version of the new and revised ISAs including the requirement to disclose KAMs in the auditor's report of public entities (IAASB 2015a, ISA 701). KAMs are selected from matters communicated with those charged with governance and that required significant auditor attention in performing the audit including including significant auditor judgments, areas of higher assessed risk of material misstatement, and the effect on the audit of significant events or transactions that occurred during the 
period. (ISA 701.9). The description of a KAM shall include an explanation of (1) why the matter is considered as strongly significant in the audit, (2) how the matter was addressed in the audit, (3) and a reference to the related disclosures in the financial statements (ISA 701.13). ISA 701 is effective for audits of financial statements of listed entities for periods ending on or after 15 December 2016.

The European Commission aspires to improve auditor reporting in a similar way. According EU-Regulation No 537/2014 the auditor's report shall provide: (1) a description of the most significant assessed risks of material misstatement, (2) a summary of the auditor's response to those risks, and (3) where relevant, key observations arising with respect to those risks. The EU-Regulation is directly applicable in all Member States and is effective for audits of public interest entities from 17 June 2016 (European Parliament and European Council of the European Union 2014).

In the UK, the FRC revised their reporting requirements already in June 2013 in order to enhance the transparency of the auditor's report aiming a better communication between auditors and users. Provisions became effective for audits of financial statements for periods commencing on or after 1 October 2012 and require auditors, among other things, to report the risks of material misstatement that had the greatest effect on: (1) the overall audit strategy, (2) the allocation of resources in the audit, and (3) directing the efforts of the engagement team (FRC 2013).

In a similar vein, the PCAOB is currently undertaking changes to the existing auditor's report, including the communication of Critical Audit Matters. Similar to KAMs, these are matters that were communicated to the audit committee and that: (1) relate to accounts or disclosures that are material to the financial statements, and (2) involved especially challenging, subjective, or complex auditor judgment. The new requirements regarding CAM disclosures will take effect for audits for fiscal years ending on or after 30 June 2019 for large accelerated filers. For all other companies to which the provisions apply, the new regulations will be effective for periods ending on or after 15 December 2020 (PCAOB 2017).

Despite the use of different terminologies, the implemented reforms of expanded auditor reporting overlap considerably. Although the requirements differ in the details, standard setters and regulators globally have clearly concluded that there is a need to disclose additional information about risk-related matters in the auditor's report. Thus, for the first time, investors gain insights into significant audit findings and procedures.

\section{Recent research on the disclosure of KAMs}

A substantial and growing body of literature investigates the effects of the disclosure of KAMs in the auditor's report. To find the relevant studies, we used different databases (for example EBSCO, Google Scholar, SSRN, Web of
Science) and searched for the key words "key audit matters" and "critical audit matters" in combination with "auditor reporting", "audit report" and comparable terms. Due to the currency of the topic, a time limitation was not necessary. Although it is common to consider only published research in literature reviews, we extended our literature review to include publicly available working papers because the majority of related studies have not yet been published. Therefore, we included working papers that have been presented at pertinent academic conferences (e.g., conferences of the American Accounting Association (AAA), the European Audit Research Network (EARNet), the International Symposium on Audit Research (ISAR)). ${ }^{2}$

The existing studies are based on experimental designs and archival data. We note that due to the lack of archival data, the majority of KAM research is experimental. Related reforms were adopted very recently, so that the only archival data for meaningful analyses is currently available from the UK, where auditors were required to report KAM since 2013.

We group the recent studies on KAM disclosures in four categories. The first category examines the effects of KAM disclosure on investor behavior and market reaction (Christensen et al. 2014; Köhler et al. 2016; Boolaky and Quick 2016; Carver and Trinkle 2017; Sirois et al. 2018; Bédard et al. 2018; Lennox et al. 2018; Gutierrez et al. 2018; Almulla and Bradbury 2018). The second category focuses on auditor responses investigating KAM effects on auditor judgement, audit fee and audit quality (Reid et al. 2018; Gutierrez et al. 2018; Almulla and Bradbury 2018; Li et al. 2018; Bédard et al. 2018; Asbahr and Ruhnke 2017, Ratzinger-Sakel and Theis 2018). The third category of papers examines whether the disclosure of KAMs will affect jurors' assessments of auditor liability (Brasel et al. 2016; Kachelmeier et al. 2018; Brown et al. 2016; Gimbar et al. 2016; Backof et al. 2018; Vinson et al. 2018). Finally, we review a handful of studies that investigate how management reporting practices are affected by (anticipated) KAM disclosures (Cade and Hodge 2014; Bentley et al. 2018; Klueber et al. 2018). Table 1 provides an overview of the recent studies about KAM disclosure.

\subsection{Investor behavior and market reaction}

Christensen et al. (2014) are among the first to demonstrate that KAM disclosures have the potential of influencing the decisions of financial statement users. They conducted an experiment among U.S. business school graduates representing nonprofessional investors and found that investors who received a KAM-like paragraph regarding the uncertainty of management estimates were more likely to stop investing in the company compared to investors who received a standard audit report (an information effect) or investors who received the same information in management's footnotes (a source credibility effect). However, they also found that the inclusion of a resolution paragraph, which contains auditor insurance for critical matters, reduces this KAM effect. 
Table 1. Summary of reviewed papers on the disclosure of KAMs (listed in alphabetical author name order).

\begin{tabular}{|c|c|c|c|c|c|}
\hline Date $^{1}$ & Author(s) ${ }^{2}$ & Method and Sample & Dependent Variable & Independent Variable & Main results \\
\hline Panel & A: Investor behavi & ior and market reaction & & & \\
\hline $\begin{array}{l}2018 \\
(w p)\end{array}$ & $\begin{array}{l}\text { Almulla/ } \\
\text { Bradbury }\end{array}$ & $\begin{array}{l}\text { Archival; New } \\
\text { Zealand; } 2015,2016, \\
\text { 2017; } 132 \text { firms }\end{array}$ & $\begin{array}{l}\text { Audit effort, audit quality, client } \\
\text { firm disclosures, investor reaction }\end{array}$ & KAM & $\begin{array}{l}\text { - Association with investor } \\
\text { uncertainty }\end{array}$ \\
\hline & Bédard/ & Archival; France, & & & $\begin{array}{l}\text { - Short-term effects: No significant } \\
\text { market reaction }\end{array}$ \\
\hline $\begin{array}{l}2018 \\
(w p)\end{array}$ & $\begin{array}{l}\text { Gonthier- } \\
\text { Besacier/Schatt }\end{array}$ & $\begin{array}{l}2002-2011 ; 1,857- \\
2,341 \text { firm-year } \\
\text { observations }\end{array}$ & $\begin{array}{l}\text { Market reaction, audit quality, } \\
\text { audit delay, audit costs }\end{array}$ & JOA & $\begin{array}{l}\text { Long-term effects: association } \\
\text { with lower agreement among } \\
\text { investors }\end{array}$ \\
\hline 2016 & Boolaky/ Quick & Experimental; 105 & Perceived financial statement & KAM, assurance level, & $\begin{array}{l}\text { - No significant effect of reporting } \\
\text { KAM or materiality level in the } \\
\text { auditor's report }\end{array}$ \\
\hline & & & & & $\begin{array}{l}\text { - But positive impact regarding the } \\
\text { disclosure of assurance level }\end{array}$ \\
\hline & & & & & $\begin{array}{l}\text { - CAMs have a negative impact on } \\
\text { readability }\end{array}$ \\
\hline 2017 & Carver/ Trinkle & $\begin{array}{l}\text { Experimental; } 150 \\
\text { non-professional }\end{array}$ & Readability, investors judgment, & CAM & $\begin{array}{l}\text { - CAMs do not influence investor's } \\
\text { valuation judgments }\end{array}$ \\
\hline & & investors & & & $\begin{array}{l}\text { However, CAMs can reduce } \\
\text { perceived management‘s } \\
\text { credibility }\end{array}$ \\
\hline 2014 & Christensen/ & $\begin{array}{l}\text { Experimental; } 141 \\
\text { Alumni from a public }\end{array}$ & Investor behavior & CAM & $\begin{array}{l}\text { Investors who receive a CAM } \\
\text { are more likely to change their } \\
\text { investment decision }\end{array}$ \\
\hline & & business school & & & $\begin{array}{l}\text { - Effect is reduced by offering a } \\
\text { resolution paragraph }\end{array}$ \\
\hline 2018 & $\begin{array}{l}\text { Gutierrez/ } \\
\text { Minutti-Meza/ } \\
\text { Tatum/Vulcheva }\end{array}$ & $\begin{array}{l}\text { Archival; UK, } \\
\text { 2011-2015, } \\
\text { 2560/2652/2056 firm- } \\
\text { year observations }\end{array}$ & $\begin{array}{l}\text { Market reaction, audit fee, audit } \\
\text { quality }\end{array}$ & $\begin{array}{l}\text { Risk of material } \\
\text { misstatement }\end{array}$ & $\begin{array}{l}\text { No significant change regarding } \\
\text { market reaction }\end{array}$ \\
\hline $\begin{array}{l}2016 \\
(w p)\end{array}$ & $\begin{array}{l}\text { Köhler/ } \\
\text { Ratzinger-Sakel/ } \\
\text { Theis }\end{array}$ & $\begin{array}{l}\text { Experimental; } 89 \\
\text { professional and } 69 \\
\text { non-professional } \\
\text { investors }\end{array}$ & Communicative value & KAM & $\begin{array}{l}\text { Higher communicative value only } \\
\text { for professional investors (no } \\
\text { communicative value for non- } \\
\text { professional investors) }\end{array}$ \\
\hline $\begin{array}{l}2018 \\
(w p)\end{array}$ & $\begin{array}{l}\text { Lennox/ } \\
\text { Schmidt/ } \\
\text { Thompson }\end{array}$ & $\begin{array}{l}\text { Archival; UK; } 2013 ; \\
488 \text { companies }\end{array}$ & Market reaction & $\begin{array}{l}\text { Risk of material } \\
\text { misstatement }\end{array}$ & $\begin{array}{l}\text { - Investors do not find disclosures } \\
\text { informative (both „short window“ } \\
\text { and „long window“ tests) }\end{array}$ \\
\hline & & & & & $\begin{array}{l}\text { Attention directing impact: users } \\
\text { pay more attention to KAM- } \\
\text { related disclosures }\end{array}$ \\
\hline 2018 & Bera & $\begin{array}{l}\text { students } \\
\text { stal }\end{array}$ & Information value & KAM & $\begin{array}{l}\text { Disclosure of several KAMs } \\
\text { leads to reduced attention towards } \\
\text { remaining parts of the financial } \\
\text { statements }\end{array}$ \\
\hline Panel & B: Auditor respon & & & & \\
\hline $\begin{array}{l}2018 \\
(w p)\end{array}$ & $\begin{array}{l}\text { Almulla/ } \\
\text { Bradbury }\end{array}$ & $\begin{array}{l}\text { Archival; } \\
\text { New Zealand; } \\
\text { 2015,2016,2017; } 32 \\
\text { firms }\end{array}$ & $\begin{array}{l}\text { Audit effort, audit quality, client } \\
\text { firm disclosures, investor reaction }\end{array}$ & KAM & $\begin{array}{l}\text { No incremental effect on audit } \\
\text { fees, audit delay or absolute } \\
\text { abnormal accruals }\end{array}$ \\
\hline $\begin{array}{l}2017 \\
(w p)\end{array}$ & Asbahr/ Ruhnke & $\begin{array}{l}\text { Experimental; } 122 \\
\text { auditors }\end{array}$ & Auditor judgment & KAM & $\begin{array}{l}\text { - No significant effect on } \\
\text { professional skepticism }\end{array}$ \\
\hline
\end{tabular}




\begin{tabular}{|c|c|c|c|c|c|}
\hline Date $^{1}$ & Author(s) $)^{2}$ & Method and Sample & Dependent Variable & Independent Variable & Main results \\
\hline \multirow{2}{*}{$\begin{array}{l}2018 \\
(w p)\end{array}$} & \multirow{2}{*}{$\begin{array}{l}\text { Bédard/ } \\
\text { Gonthier- } \\
\text { Besacier/ Schatt }\end{array}$} & \multirow{2}{*}{$\begin{array}{l}\text { Archival; France, } \\
\text { 2002-2011; 1,857- } \\
2,341 \text { firm-year } \\
\text { observations }\end{array}$} & \multirow{2}{*}{$\begin{array}{l}\text { Market reaction, audit quality, } \\
\text { audit delay, audit costs }\end{array}$} & \multirow{2}{*}{ JOA } & $\begin{array}{l}\text { - Short-term effects: positive } \\
\text { association with audit lag and } \\
\text { audit fees }\end{array}$ \\
\hline & & & & & $\begin{array}{l}\text { Long-term effects: association } \\
\text { with lower agreement among } \\
\text { investors and reporting quality }\end{array}$ \\
\hline 2018 & $\begin{array}{l}\text { Gutierrez/ } \\
\text { Minutti-Meza/ } \\
\text { Tatum/ Vulcheva }\end{array}$ & $\begin{array}{l}\text { Archival; UK, } \\
2011-2015, \\
\text { 2560/2652/2056 firm- } \\
\text { year observations }\end{array}$ & $\begin{array}{l}\text { Market reaction, audit fee, audit } \\
\text { quality }\end{array}$ & $\begin{array}{l}\text { Risk of material } \\
\text { misstatement }\end{array}$ & $\begin{array}{l}\text { - No significant change regarding } \\
\text { audit fee and audit quality }\end{array}$ \\
\hline $\begin{array}{l}2018 \\
(w p)\end{array}$ & Li/ Hay/ Lau & $\begin{array}{l}\text { Archival; New } \\
\text { Zealand; } 2016 ; \\
\text { 182/242 firm-year } \\
\text { observations }\end{array}$ & Audit quality, Audit fees & KAM & $\begin{array}{l}\text { - Improvement of audit quality } \\
\text { accompanying with an increase in } \\
\text { audit fees }\end{array}$ \\
\hline $\begin{array}{l}2018 \\
(w p)\end{array}$ & $\begin{array}{l}\text { Ratzinger-Sakel/ } \\
\text { Theis }\end{array}$ & $\begin{array}{l}\text { Experimental; } 73 \\
\text { auditors }\end{array}$ & Auditor judgment performance & KAM & $\begin{array}{l}\text { Less professional skepticism } \\
\text { when KAM consideration is } \\
\text { present }\end{array}$ \\
\hline \multirow{2}{*}{$\begin{array}{l}2018 \\
(w p)\end{array}$} & \multirow{2}{*}{$\begin{array}{l}\text { Reid/ Carcello/ } \\
\text { Li/ Neal }\end{array}$} & \multirow{2}{*}{$\begin{array}{l}\text { Archival; UK; } 1088 \\
(888,884) / 1304 / \\
1292 \text { firm-year } \\
\text { observations }\end{array}$} & \multirow{2}{*}{$\begin{array}{l}\text { Financial reporting quality, audit } \\
\text { fee, audit delay }\end{array}$} & \multirow{2}{*}{$\begin{array}{l}\text { Risk of material } \\
\text { misstatement }\end{array}$} & $\begin{array}{l}\text { Significant improvement in } \\
\text { financial reporting quality }\end{array}$ \\
\hline & & & & & $\begin{array}{l}\text { - No effect on audit fee and audit } \\
\text { delay }\end{array}$ \\
\hline
\end{tabular}

Panel C: Auditor liability

\begin{tabular}{|c|c|c|c|c|c|}
\hline \multirow{2}{*}{$\begin{array}{l}2018 \\
(w p)\end{array}$} & \multirow{2}{*}{$\begin{array}{l}\text { Backof/ Bowlin/ } \\
\text { oodson }\end{array}$} & \multirow{2}{*}{$\begin{array}{l}\text { Experimental; } 63 \\
\text { undergraduate } \\
\text { students }\end{array}$} & \multirow[t]{2}{*}{ Auditor liability } & \multirow[t]{2}{*}{ CAM } & $\begin{array}{l}\text { - When the audit report includes } \\
\text { a related CAM disclosure, } \\
\text { jurors perceive auditors as more } \\
\text { negligent }\end{array}$ \\
\hline & & & & & $\begin{array}{l}\text { - However, clarifying the concept } \\
\text { of reasonable assurance mitigates } \\
\text { this effect }\end{array}$ \\
\hline 2016 & $\begin{array}{l}\text { Brasel/ Doxey/ } \\
\text { Grenier/ Reffett }\end{array}$ & $\begin{array}{l}\text { Experimental; } 528 \\
\text { participants from } \\
\text { Amazon Mechanical } \\
\text { Turk }\end{array}$ & Auditor liability & CAM & $\begin{array}{l}\text { - CAMs reduce jurors` auditor } \\
\text { liability judgments under certain } \\
\text { conditions (but only if undetected } \\
\text { misstatements are, absent CAM } \\
\text { disclosure, relatively difficult to } \\
\text { foresee) }\end{array}$ \\
\hline $\begin{array}{l}2016 \\
(w p)\end{array}$ & $\begin{array}{l}\text { Brown/ Majors/ } \\
\text { Peecher }\end{array}$ & $\begin{array}{l}\text { Experimental; } 239 \\
\text { participants from } \\
\text { Amazon Mechanical } \\
\text { Turk and } 116 \text { law } \\
\text { students }\end{array}$ & Auditor liability & $\begin{array}{l}\text { CAM (only as } \\
\text { a supple-mental } \\
\text { manipulation) }\end{array}$ & $\begin{array}{l}\text { - No significant main effect of } \\
\text { CAMs on liability judgments }\end{array}$ \\
\hline \multirow{2}{*}{2016} & \multirow{2}{*}{$\begin{array}{l}\text { Gimbar/ } \\
\text { Hansen/ } \\
\text { Ozlanski }\end{array}$} & \multirow{2}{*}{$\begin{array}{l}\text { Experimental; } 234 \\
\text { students }\end{array}$} & \multirow{2}{*}{ Auditor liability } & \multirow{2}{*}{ CAM } & $\begin{array}{l}\text { - Under precise standards, both } \\
\text { related and unrelated CAMs } \\
\text { increase auditor liability }\end{array}$ \\
\hline & & & & & $\begin{array}{l}\text { - CAMs increase auditor liability by } \\
\text { a lesser amount under imprecise } \\
\text { standards than precise standards }\end{array}$ \\
\hline \multirow[t]{2}{*}{$\begin{array}{l}2018 \\
(w p)\end{array}$} & \multirow[t]{2}{*}{$\begin{array}{l}\text { Kachelmeier/ } \\
\text { Schmidt/ } \\
\text { Valentine }\end{array}$} & \multirow[t]{2}{*}{$\begin{array}{l}\text { Experimental; } 70 \\
\text { attorneys, } 50 \text { financial } \\
\text { analysts and } 150 \mathrm{MBA} \\
\text { students }\end{array}$} & \multirow[t]{2}{*}{ Auditor legal exposure } & \multirow[t]{2}{*}{ CAM } & $\begin{array}{l}\text { - CAM disclosure decreases } \\
\text { assessments of auditor } \\
\text { responsibility when the } \\
\text { misstatement is in the same area } \\
\text { as the CAM }\end{array}$ \\
\hline & & & & & $\begin{array}{l}\text { - "Disclaimer effect" is manifest in } \\
\text { different ways for different groups }\end{array}$ \\
\hline
\end{tabular}




\begin{tabular}{|c|c|c|c|c|c|}
\hline Date $^{1}$ & Author(s) ${ }^{2}$ & Method and Sample & Dependent Variable & Independent Variable & Main results \\
\hline \multirow[b]{2}{*}{$\begin{array}{l}2018 \\
(w p)\end{array}$} & \multirow{2}{*}{$\begin{array}{l}\text { Vinson/ } \\
\text { Robertson/ } \\
\text { Cockrell }\end{array}$} & \multirow{2}{*}{$\begin{array}{l}\text { Experimental; } 168 \\
\text { participants from } \\
\text { Amazon Mechanical } \\
\text { Turk }\end{array}$} & \multirow[b]{2}{*}{ Auditor liability } & \multirow[b]{2}{*}{ CAM } & $\begin{array}{l}\text { - Higher auditor negligence when a } \\
\text { CAM is removed }\end{array}$ \\
\hline & & & & & $\begin{array}{l}\text { Highest assessed negligence when } \\
\text { auditor removes a CAM after } \\
\text { reporting it for multiple years }\end{array}$ \\
\hline \multicolumn{6}{|c|}{ Panel D: Client management responses } \\
\hline \multirow[t]{2}{*}{$\begin{array}{l}2018 \\
(w p)\end{array}$} & \multirow[t]{2}{*}{$\begin{array}{l}\text { Bentley/ } \\
\text { Lambert/ Wang }\end{array}$} & \multirow[t]{2}{*}{$\begin{array}{l}\text { Experimental; } 140 \\
\text { corporate managers }\end{array}$} & \multirow[t]{2}{*}{ Manager's decision making } & \multirow[t]{2}{*}{ CAM } & $\begin{array}{l}\text { - Given a Standard CAM, managers } \\
\text { were less likely to hedge (a } \\
\text { risk-decreasing transaction), but } \\
\text { more likely to speculate (a risk- } \\
\text { increasing transaction) }\end{array}$ \\
\hline & & & & & $\begin{array}{l}\text { A Disclaimer CAM mitigates the } \\
\text { impact of CAM on speculation }\end{array}$ \\
\hline $\begin{array}{l}2014 \\
(w p)\end{array}$ & Cade/ Hodge & Experimental; Alumni & $\begin{array}{l}\text { Communication between } \\
\text { management and auditors }\end{array}$ & Additional disclosures & $\begin{array}{l}\text { Managers are less willing to share } \\
\text { accounting choices with auditors }\end{array}$ \\
\hline $\begin{array}{l}2018 \\
(w p)\end{array}$ & $\begin{array}{l}\text { Klueber/ Gold/ } \\
\text { Pott }\end{array}$ & $\begin{array}{l}\text { Experimental; } 54 \\
\text { participants }\end{array}$ & Manager's decision making & KAM & $\begin{array}{l}\text { - Reduced earnings management } \\
\text { if KAM section includes firm- } \\
\text { specific information }\end{array}$ \\
\hline
\end{tabular}

1 "wp" indicates that a paper is not yet published at the time of writing this review.

2 The articles are listed in alphabetical order of the author names.

Köhler et al. (2016) also undertook an experiment to examine the communicative value of the expanded auditor's report among professional and non-professional investors for a sample that consisted primarily of German users. They find that investment professionals' assessments of the economic situation of a company are influenced by variations in the KAM disclosures. However, KAM disclosures appear to have no communicative value on non-professional investors as they may have difficulties to process the new information revealed by KAM. Another German study (Boolaky and Quick 2016) focuses on yet another financial statement user group, that of bank directors. The authors examine the effect of KAM disclosure on bank directors' perceptions of financial reporting quality and credit approval decisions, but found no effect of KAM disclosure.

An experimental study by Carver and Trinkle (2017) examines the impact of KAM disclosure on nonprofessional investors' perceptions of audit report readability, their valuation judgments, and their evaluations of management's credibility. Findings suggest that KAM disclosures lead to a less readable report that did not result in incremental changes of investors' valuation judgments (neither directly nor through its effect on readability). However, they found a negative impact of KAM disclosure on investors' perceptions of management's credibility when earnings just meet analyst's forecasts.

The experimental study by Sirois et al. (2018) provides interesting insights into how users' information search strategies are affected. The authors asked graduate accounting students in Canada to assume the role of bank loan officer and examined the influence of KAMs on users' attention to financial statement information. Using innovative eye-tracking technology, the researchers found that KAMs have an attention-directing effect, such that KAMs increase users' attention to KAM-related information in the financial statement disclosures. Moreover, the presence of KAMs leads to a reduction of the level of attention devoted to parts of the financial statements not covered by the KAMs, indicating that KAMs have the potential of helping investors effectively navigate through the financial report and to focus their attention on pertinent issues.

While most research thus far is based on experiments, there is also some initial evidence from archival studies. First, Bédard et al. (2018) investigate the effects of "justifications of assessment (JOAs)" in the French setting. Since 2003, auditors in France have been required to disclose items important to the understanding of the financial report. As these disclosures include a summary of auditor's assessments, performed procedures and a conclusion, the research results are comparable to KAM settings and thus relevant for our objective. Bédard et al. (2018) do not observe a significant market reaction to disclosure of first JOAs, but subsequent disclosure of JOAs was significantly associated with larger abnormal trading volume (i.e., lower agreement among investors).

Lennox et al. (2018) examine the expanded UK reporting model. Using short-window and long-window tests, the authors investigate market reactions following risk disclosure in the auditor's report to assess whether investors perceive the new disclosures as informative. Their results suggest that the new disclosures were reliable but that they lack of incremental information content because users were already informed about the majority of the risks before these risks were reported in the auditor's report.

Gutierrez et al. (2018) perform an archival study to examine the consequences of additional information in 
the auditor's report on investor's reaction, audit fees and audit quality. Implementing a difference-in-difference research design, they do not find evidence for an incremental short market reaction. Their results regarding investor's reaction (measured by abnormal returns and abnormal trading volume) align with the findings of Lennox et al. (2018) concluding that KAM disclosure does not influence investor behavior.

In contrast to the above archival studies, Almulla and Bradbury (2018) find that KAMs are associated with investor uncertainty. Interestingly, examining the first year of KAM disclosures in New Zealand, they observe that investors already valued the risks in the year before KAM disclosure was implemented.

Overall, the above studies provide mixed results regarding investor behavior and market reaction in response to KAMs. Some experimental studies suggest that there is an effect on users, showing that users are less likely to invest in a company and that they focus their attention on particular parts of the financial statements in the presence of KAM disclosure. However, other experimental results do not confirm these effects and, importantly, archival research has not been able to find evidence in support of a significant market reaction. In view of these mixed results, further research is necessary to explore the economic consequences associated with KAM disclosure. In particular, archival research will be feasible once regulatory developments in other jurisdictions take effect and sufficient data is available.

\subsection{Auditor responses}

While the impact of KAMs on reducing the information gap and thus investor behavior is most directly aligned with the intended objectives of the expanded audit reporting model, some researchers have also examined whether the requirement to disclose KAMs influences auditor behavior. According to Reid et al. (2018), the audit may be affected by KAM disclosure because (1) management may adopt a more acceptable accounting behavior due to the threat of auditor disclosure, and (2) auditors may feel more accountable for their work and therefore do a better job. There are several working papers that offer initial evidence of the association between audit report expansion and audit-related outcomes.

First, while not focusing exclusively on disclosure of KAMs, Reid et al. (2018) is one of the first studies to examine the relationship between the new reporting regime (which includes KAM disclosure) and audit-related outcomes. They focus on the UK and find that the new reporting regime leads to significant improvement in financial reporting quality (as proxied by absolute abnormal accruals, the propensity to just meet or beat analyst forecasts, and a significant increase in earnings response coefficients) without detecting a significant increase in audit costs (neither fees or audit delays). While these findings may be driven by other elements of the new reporting model, the study provides initial evidence of beneficial effects of KAMs for audit quality.
Gutierrez et al. (2018) also focus on the UK experience but report slightly different results with respect to audit-related outcomes. They observe no significant association between the expanded auditor's report and either audit fees or audit quality. Initial evidence from New Zealand offers inconsistent insights. First, Almulla and Bradbury (2018) find no incremental effect of the expanded auditor's report on either audit fees, audit delay or absolute abnormal accruals. In contrast, Li et al. (2018) report that the introduction of the new and revised audit reporting standards were followed by an improvement in audit quality (as proxied by a reduction in absolute abnormal accruals) and a significant increase in audit fees, suggesting that although the new auditor reporting model results in audit quality improvements, such benefit comes at a cost.

Bédard et al. (2018) focus more directly on disclosure of JOAs in the French setting, which, as discussed, are similar to KAMs. They find negative efficiency effects in the first year of disclosure (i.e., longer audit report lag and increased audit fees), but not in subsequent years. Interestingly, in subsequent years (but not the first year) the disclosure of JOAs is negatively associated with financial reporting quality (as proxied by discretionary accruals). While these findings are inconsistent with the results reported by Reid et al. (2018) and Gutierrez et al. (2018), the authors argue that they could also be explained by the fact that clients for which JOAs are disclosed are subject to accounting information that is more difficult to audit and thus measurement error and bias are more likely in these cases.

While these studies provide important preliminary archival evidence, additional research over more years and in other jurisdictions will help better reconcile the longer-term effects. Finally, we are aware of two working papers that use the experimental method to examine how auditors in Germany respond to the (anticipated) disclosure of KAMs (Asbahr and Ruhnke 2017; Ratzinger-Sakel and Theis 2018). Interestingly, both studies suggest that auditors that are asked to consider KAMs exhibit less professional skepticism than when they do not consider KAMs, suggesting adverse effects of KAMs on auditor judgment performance.

Again, we conclude that the evidence with respect to the association between KAM disclosure and audit-related outcomes is mixed, but we note that surprisingly many studies suggest adverse effects, which require deeper investigation to be corroborated and analyzed.

\subsection{Auditor liability}

In the course of the development of the new reporting requirement auditor legal liability was a frequently debated controversy, particularly in the United States (e.g., Tysiac 2013). According to some, disclosing KAMs might increase jurors' auditor liability judgments when auditors have failed to detect misstatements. As a result, a third stream of research examines experimentally whether KAM dis- 
closure influences liability judgments. Typically using jury-eligible individuals as participants, some studies have found that disclosing KAMs can actually reduce auditor liability (Brasel et al. 2016; Kachelmeier et al. 2018) or have no effect (Brown et al. 2016), suggesting that the concern over the legal hazards of disclosing KAMs is likely unwarranted. Other studies have found that KAMs have the potential of increasing liability. For instance, Gimbar et al. (2016) find that KAMs increase auditor liability relative to the traditional audit report, albeit to a lesser degree under imprecise standards. Similarly, Backof et al. (2018) find that reporting a KAM increases jurors' negligence assessments, but explaining the concept of reasonable assurance mitigates this effect. Vinson et al.'s (2018) experiment considers longer-term effects of KAM disclosure. They find that removing a KAM that is reported for multiple years, relative to a KAM that is reported for one year, results in higher negligence assessments due to higher perceptions that the misstatement was foreseeable to the auditor.

\subsection{Client management responses}

A few working papers have examined whether and how client management responds to (anticipated) KAM disclosures by their auditors. It is possible that KAMs may influence managerial decision making, given the increased scrutiny by auditors as a result of KAMs. We are aware of three experimental studies which are relevant in this regard. First, Cade and Hodge (2014) investigate whether KAM-like details in the auditor's report affect how openly managers communicate with their auditors. Interestingly, they find that managers share less private information with their auditors about their accounting choices when they are told that the auditor will publicly disclose such choices, a potentially adverse effect of the KAM regime. Anticipating auditors' disclosure of audit procedures does not have such adverse effects.

Bentley et al. (2018) investigate whether the anticipation of KAM-like auditor disclosures affect managerial decision-making and find that managers are more likely to speculate and less likely to hedge when they anticipate a KAM disclosure. This effect is mitigated when the KAM report contains a disclaimer related to the scope of the auditor's assurance role.

Finally, Klueber et al. (2018) ask managers about their financial reporting choices and examine whether earnings management is reduced as a result of anticipated KAM disclosure. They find that as long as the KAM section includes firm-specific information, it indeed has the potential of reducing earnings management in financial reporting.

\section{Conclusions, implications and suggestions for future research}

Responding to extensive criticism of the traditional pass/ fail-model of auditor reporting, standard setters and regulators worldwide have recently released new auditor reporting requirements, including the requirement for auditors to dis- close Key Audit Matters (KAMs). The disclosure of KAMs is supposed to enhance the information value and decision usefulness of the auditor's report, and may also have effects on the performance of auditors and managers, as well as liability judgments of jurors. We identified 22 research studies examining the consequences of KAM disclosures for investor behavior, auditor responses, jurors' assessments of auditor liability, and client management responses.

Several research findings support the intended benefits of KAM disclosures. For example, experimental evidence suggests that KAMs have the potential of influencing the decisions of financial statement users, particularly with regard to non-professional investors. KAMs also have the potential of effectively directing financial statement users' attention to pertinent areas and decrease managers' earnings management attempts. While these findings are promising, preliminary archival research fails to support a wider capital market reaction to KAM disclosures, raising questions about the economic significance of the changed reporting model.

KAM disclosures also appear to have some unanticipated, and sometimes even adverse consequences. Archival research finds efficiency losses in terms of increased audit report lags and audit fees, and experimental evidence suggests that auditors may be less professionally skeptical in the presence of KAMs. Finally, managers' willingness to share information with their auditor as well as their risk-taking behavior is affected by anticipated KAM disclosures, not always in a beneficial direction.

Based on the findings reviewed in this paper, we offer some important implications and recommendations for audit practice. First, auditors should be aware that the disclosure of KAMs has attention-directing impacts on financial statement users and should therefore carefully decide how many, and in particular what matters, they disclose as KAMs in the auditor's report. Second, some adverse consequences of KAM disclosures can be mitigated by an explanation of the concept of reasonable assurance (e.g., Backof et al. 2018) and providing information specificity in KAMs (e.g., Klueber et al. 2018). As a result, standard setters may consider prescribing more clarifying language in KAM disclosures. In general, standard setters and regulators should pay close attention to adverse effects that KAM disclosures may have in the coming years.

In a relatively short time span, a substantial number of research papers has appeared with the objective of examining the consequences of KAM disclosures for a variety of stakeholders, suggesting this is a growing body of auditing research. The findings thus far suggest several fruitful avenues for future research. In particular, the mixed nature of results of previous studies indicate that there may be insufficient research to assess all the consequences of KAM disclosure. First, due to the mixed findings on investor and market reactions, more research is needed on how exactly investors process the additional information provided in KAM sections, while differentiating between sophisticated and unsophisticated investors. In this regard, we recommend greater use of qualitative research methods (e.g., interviews or focus 
groups) to better understand how users process this information. Eye-tracking research, such as the study by Sirois et al. (2018) may also provide additional insights into such processes. Second, as data becomes available in ISA jurisdictions, UK archival studies should be replicated to make comparisons between different jurisdictions. As ISA 701 is mandatory for audits for periods ending on or after 15 December 2016, further archival research can be expected soon; and the emerging CAMs in the United States will also offer plenty of research opportunities. Finally, future studies could focus on the production process of KAMs which has not yet been considered in prior research and which may also have (indirect) effects on various reporting and quality outcomes. For example, future field studies could examine the process used to identify and select KAMs and how this process influences auditor communications with management and audit committees.

- Prof. dr. A. Gold is full professor of auditing at Vrije Universiteit Amsterdam and adjunct professor at Norwegian School of Economics (NHH)

- M. Heilmann MSc is a Ph.D. student at Technische Universität Dortmund

\section{Notes}

1. In addition to the disclosure of KAMs, regulatory developments also include other changes to the content and the form of the auditor's report. However, our literature review focuses on studies examining the impact of KAM disclosures only.

2. We caution readers that findings and conclusions reported in working papers may change as a result of the academic review process, which we however consider a relatively minor trade-off to our choice to include working papers in our review.

\section{References}

- Almulla M, Bradbury ME (2018) Auditor, client, and investor consequences of the enhanced auditor's report. Working paper. https:// papers.ssrn.com/sol3/papers.cfm?abstract_id=3165267

- Asare SK, Wright AM (2012) Investors', auditors', and lenders' understanding of the message conveyed by the standard audit report on the financial statements. Accounting Horizons 26(2): 193-217. https://doi.org/10.2308/acch-50138

- Asbahr K, Ruhnke K (2017) Real effects of reporting key audit matters on auditors' judgment of accounting estimates. Working paper. https://doi.org/10.2139/ssrn.3069755

- Backof AG, Bowlin K, Goodson B (2018) The importance of clarification of auditors' responsibilities under the new audit reporting standards. Working paper. https://papers.ssrn.com/sol3/papers. cfm?abstract_id $=2446057$

- Bédard J, Gonthier-Besacier N, Schatt A (2018) Consequences of justifications of assessments in French expanded audit reports. Working paper. https://doi.org/10.2139/ssrn.3175497

- Bentley JW, Lambert TA, Wang E (2018) The effect of increased audit disclosure on managerial decision making: Evidence from disclosing critical audit matters. Working paper. https://papers.ssrn. com/sol3/papers.cfm?abstract_id $=3000978$

- Boolaky PK, Quick R (2016) Bank directors' perceptions of expanded auditor's reports. International Journal of Auditing 20: 158-174. https://doi.org/10.1111/ijau.12063

- Brasel K, Doxey MM, Grenier JH, Reffett A (2016) Risk disclosure preceding negative outcomes: The effects of reporting critical audit matters on judgments of auditor liability. The Accounting Review 91(5): 1345-1362. https://doi.org/10.2308/accr-51380

- Brown T, Majors TM, Peecher ME (2016) The impact of a higher intent standard on auditors' legal exposure and the moderating role of jurors' legal knowledge. Working paper. https://papers.ssrn.com/ sol3/papers.cfm?abstract_id $=2483221$
- Cade N, Hodge F (2014) The effect of expanding the audit report on managers' communication openness. Working paper. https://papers. ssrn.com/sol3/papers.cfm?abstract_id=2433641

- Carcello JV (2012) What do investors want from the standard audit report? Results of a survey of investors conducted by the PCAOB's Investor Advisory Group. The CPA Journal 82(1): 22-28.

- Carver BT, Trinkle BS (2017) Nonprofessional investors' reactions to the PCAOB's proposed changes to the standard audit report. Working paper. https://papers.ssrn.com/sol3/results.cfm?RequestTimeout $=50000000$

- Chong K-M, Pflugrath G (2008) Do different audit report formats affect shareholders' and auditors' perceptions? International Journal of Auditing 12(3): 221-241. https://doi.org/10.1111/j.10991123.2008.00381.x

- Christensen BE, Glover SM, Wolfe CJ (2014) Do critical audit matter paragraphs in the audit report change nonprofessional investors' decision to invest? Auditing, A Journal of Practice \& Theory 33(4): 71-93. https://doi.org/10.2308/ajpt-50793

- Church BK, Davis SM, McCracken SA (2008) The auditor's reporting model: A literature overview and research synthesis. Accounting Horizons 22(1): 69-90. https://doi.org/10.2308/acch.2008.22.1.69

- European Parliament and Council of the European Union (2014) Regulation of the European Parliament and of the Council on Specific Requirements Regarding Statutory Audit of Public-Interest Entities and Repealing Commission Decision 2005/909/EU. Brussels: European Parliament and Council of the European Union. https://eur-lex. europa.eu/legal-content/EN/TXT/?uri=celex:32014R0537

- FRC (Financial Reporting Council), International Standard on Auditing (UK and Ireland) 700 (2013) The independent auditor's report on financial statements. https://www.frc.org.uk/getattachment/501de004-b616-43c3-8d65-aeaebde19f8d/ISA-700-(UK-andIreland)-700-(Revised)-Independent-auditors-report-June-2013.pdf 
- Gimbar C, Hansen B, Ozlanski ME (2016) The effects of critical audit matter paragraphs and accounting standard precision on auditor liability. The Accounting Review 91(6): 1629-1646. https://doi. org/10.2308/accr-51382

- Gold A, Gronewold U, Pott C (2012) The ISA 700 auditor's report and the audit expectation gap - Do explanations matter? International Journal of Auditing 16(3): 286-307. https://doi.org/10.1111/ j.1099-1123.2012.00452.x

- Gray GL, Turner JL, Coram PJ, Mock TJ (2011) Perceptions and misperceptions regarding the unqualified auditor's report by financial statement preparers, users, and auditors. Accounting Horizons 25(4): 659-684. https://doi.org/10.2308/acch-50060

- Gutierrez E, Minutti-Meza M, Tatum KW, Vulcheva M (2018) Consequences of adopting an expanded auditor's report in the United Kingdom. Review of Accounting Studies, 23: 1543-1587. https:// doi.org/10.1007/s11142-018-9464-0

- IAASB (International Auditing and Assurance Standards Board) (2011) Enhancing the value of auditor reporting: Exploring options for change. https://www.ifac.org/system/files/publications/exposure-drafts/CP_Auditor_Reporting-Final.pdf

- IAASB (International Auditing and Assurance Standards Board) (2012) Invitation to comment: Improving the auditor's report. http://www.ifac.org/system/files/publications/files/Auditor_Reporting_Invitation_to_Comment-final_0.pdf

- IAASB (International Auditing and Assurance Standards Board) (2013) Reporting on audited financial statements: Proposed new and revised International Standards on Auditing (Exposure Draft). https://www.ifac.org/publications-resources/reporting-audited-financial-statements-proposed-new-and-revised-international

- IAASB (International Auditing and Assurance Standards Board) (2015a) International Standard on Auditing (ISA) 701 Communicating Key Audit Matters in the Independent Auditor's Report. https:// www.ifac.org/publications-resources/international-standard-auditing-isa-701-new-communicating-key-audit-matters-i

- IAASB (International Auditing and Assurance Standards Board) (2015b) At a glance: New and revised auditor reporting standards and related conforming amendments. http:/www.ifac.org/system/files/ uploads/IAASB/Audit\%20Reporting-At\%20a\%20Glance-final.pdf

- Kachelmeier SJ, Schmidt JJ, Valentine K (2018) Do critical audit matter disclosures protect auditors by forewarning users of misstatement risk? Working paper, available at: https://papers.ssrn.com/sol3/ papers.cfm?abstract_id $=2481284$

- Klueber J, Gold A, Pott C (2018) Do key audit matters impact financial reporting behavior? Working paper. https://papers.ssrn.com/ sol3/papers.cfm?abstract_id $=3210475$

- Köhler AG, Ratzinger-Sakel NVS, Theis JC (2016) The effects of key audit matters on the auditor's report's communicative value: Experimental evidence from investment professionals and non-professional investors. Working paper. https://doi.org/10.2139/ssrn.2838162
- Lennox CS, Schmidt JJ, Thompson AM (2018) Is the expanded model of audit reporting informative to investors? Evidence from the U.K. Working paper. https://papers.ssrn.com/sol3/papers. cfm?abstract_id=2619785

- Li H, Hay D, Lau D (2018) Assessing the impact of the new auditor's report. Working paper. https://doi.org/10.2139/ssrn.3120822

- Mock TJ, Bédard J, Coram PJ, Davis SM, Espahbodi R, Warne RC (2013) The audit reporting model: Current research synthesis and implications. A Journal of Practice \& Theory 32(1): 323-351. https://doi.org/10.2308/ajpt-50294

- PCAOB (Public Company Accounting Oversight Board) (2017) The auditor's report on an audit of financial statements when the auditor expresses an unqualified opinion. https://pcaobus.org/Rulemaking/ Docket034/2017-001-auditors-report-final-rule.pdf

- Prasad P, Chand P (2017) The changing face of the auditor's report: Implications for suppliers and users of financial statements. Australian Accounting Review 83(27): 348-367. https://doi.org/10.1111/ auar. 12137

- Ratzinger-Sakel NVS, Theis JC (2018) Does considering key audit matters affect auditor judgment performance? https://papers.ssrn. com/sol3/papers.cfm?abstract_id=3003318

- Reid LC, Carcello JV, Li C, Neal TL (2018) Impact of auditor report changes on financial reporting quality and audit costs: Evidence from the United Kingdom. Working paper. https://papers.ssrn.com/ sol3/papers.cfm?abstract_id $=2647507$

- Simnett R, Huggins A (2014) Enhancing the auditor's report: To what extent is there support for the IAASB's proposed changes? Accounting Horizons 28(4): 719-747. https://doi.org/10.2308/ acch-50791

- Sirois L-P, Bédard J, Bera P (2018) The informational value of key audit matters in the auditor's report: Evidence from an eye-tracking study. Accounting Horizons 32(2): 141-162. https://doi. org/10.2308/acch-52047

- Turner JL, Mock TJ, Coram PJ, Gray GL (2010) Improving transparency and relevance of auditor communications with financial statement users. Current Issues in Auditing 4(1): A1-A8. https://doi. org/10.2308/ciia.2010.4.1.A1

- Tysiac K (2013) PCAOB's reporting model proposal poses risks, rewards for audit firms. Journal of Accountancy, August 23. https://www. journalofaccountancy.com/news/2013/aug/20138576.html

- Vanstraelen A, Schelleman C, Meuwissen R, Hofmann I (2012) The audit reporting debate: Seemingly intractable problems and feasible solutions. European Accounting Review 21(2): 193-215. https://doi. org/10.1080/09638180.2012.687506

- Vinson JM, Robertson JC, Cockrell RC (2018) The effects of critical audit matter removal and duration on jurors' assessments of auditor negligence. Working paper. https://doi.org/10.2308/ajpt52319 